\title{
Geomagnetic Control of Universal Variation of $f_{\mathrm{o}} F 2$
}

\author{
V. V. Kuznetsov, V. V. Plotkin, I. I. Nesterova, and M. S. Pozdeeva \\ Institute of Geophysics, Siberian Branch, Russian Academy Science, Novosibirsk, Russia
}

(Received April 21, 1994; Revised November 26, 1994; Accepted Decembcr 27, 1994)

\begin{abstract}
There is improved the model for the description of the longitude-temporal variations of the $F 2$-layer critical frequency which is based on the notion about the universal variation. This variation is characterized by the united change of $f \circ F 2$ at the Universal Time (UT) at all longitudes of the given latitude ring. In this paper the temporal and longitudinal dependence of the universal component amplitude is investigated. It was done by the selection of the best approximation of the observed $f \circ F 2$-variations by means of the model in the form of the sum of the local and universal components.
\end{abstract}

\section{Introduction}

The diurnal variations of the ionospheric parameters are subjected to the essential control by the solar local time of the observatory. It is explained by the substantial influence of the Solar radiation on the ionization of the ionosphere. In a large measure it defines the latitudinal dependence of the ionospheric parameters.

However the diurnal behaviour of the ionospheric parameters depends essentially not only from the observatory latitude. It exhibits also the longitudinal variations. The longitudinal effect of the $F 2$-layer critical frequency was first mentioned in the literature (Kessenikh and Bulatov, 1944; Appleton, 1946; Bailey, 1948). The notion on the longitudinal effect have arised owing to using the method of the data processing. When the data of the ground ionospheric sounding were used, the longitudinal dependence was usually studied for the midday magnitudes of $f \circ F 2$ at the zone time. Note that it is more correct to analyse the data for the midday according to the solar local time.

Later the longitudinal dependences were studied not only for the midday magnitudes, especially when it became possible to obtain the $f$ o F2-magnitudes on the satellites (Rastogi, 1963; Tulunay, 1973; Khachikyan and Rudina, 1979; Kochenova, 1987). The limitation of the satellite's data is, in our opinion, the deficiency of the synchronous observations of $f_{0} F 2$ for the different moments of the local time (LT). The long time is necessary to gather the data for such analysis by alone satellite. The world network of the ground ionosondes gives the chance to obtain the ionospheric parameters simultaneously in some points with the different LT. Therefore in this paper we study these effects analysing the ground data.

The diurnal behaviour at the observatories with the different longitude is measured at the different UT, therefore it is possible to say also about UT-control of the ionosphere condition (Kohnlein and Raitt, 1978; Osipov and Maksimova, 1983; Besprozvannaya and Makarova, 1984; Maksimova and Osipov, 1984). Note that we keep in mind only the quiet condition but not the storms or another UT-disturbances.

It was found that the maxima of the longitudinal dependences of $f \circ F 2$ for the different LT occur at the different longitudes, but all of them occur at the same UT. It is clearly seen in the Southern hemisphere data where the $f \circ F 2$-maximum at middle latitudes is observed at $07 \mathrm{UT}$ (Eccles et al., 1971; Kohnlein and Raitt, 1978). The similar UT-effects were found at the high latitude stations when the diurnal behaviour of the sun-lighting was not essential (Kneht, 1959; Duncan, 1962; King et al., 1968; Challinor, 1970; Hopkins et al., 1973). These facts led to the notion about UT-control of the ionosphere. Deminov and Karpachev (1986) connect the longitudinal control of the ionosphere with the existence of the world magnetic anomalies and they connect the UT-control with the geographical and geomagnetic axes 
uncoincidence. In doing so they have noted the conventionality of such separation.

In essence, we are dealing with the terminology. An important point is that besides the solar radiation it is necessary to take into account the other physical factors having influence on the ionosphere condition, for example, the meteorological effects (Kazimirovsky et al., 1983), the neutral-air winds (Challinor and Eccles, 1971; Eccles et al., 1971), the magnetic declination (Eyfrig, 1963), the electric field in the ionosphere (Pancheva, 1986) and the electric field in the atmosphere near the Earth's surface (Kuznetsov et al., 1992, 1993). In all these cases we are dealing with the plasma processes controlled by the geomagnetic field. Therefore one expect that the longitudinal differences in the diurnal behaviour are caused by the peculiarities of the magnetic field structure: firstly the geographical and geomagnetic axes do not coincide, besides there are the world magnetic anomalies. The latter causes the original structure of the Northern and Southern magnetic poles of the Earth. We would like to note that 07 UT and 19 UT are the instants of time when during the diurnal rotation the local midday or the local midnight comes in the regions of the Earth's magnetic poles (Schweidler, 1932). In this connection it should be interest to clear up the peculiarities of the longitude-temporal variations of the ionospheric characteristics connected with the mentioned circumstances.

In this paper we want to show the existence of the geomagnetic control of the universal variation $f \circ F 2$ (Kuznetsov et al., 1990). This variation was defined by us as UT-variation of the mean-longitude value $f$ o $F 2$. It was called as the universal variation by analogy with the known variation of the gradient of the electrical potential near the Earth's surface (Chalmers, 1967). Now we would like to extend our notion about the universal variation of the geophysical parameters and, in particular, the magnitude $f \circ F 2$.

2. Preliminary Analysis of Relation between the Longitudinal and UT-Variations of $f \circ F 2$ at MidLatitudes

When studying the geophysical fields in the near Earth space it is convenient to use both the usual geographical system of coordinates which is fixed relatively the Earth and the coordinate system which is rotating around the same axis $\mathrm{OZ}$ and is fixed relatively the Sun. The latter is named here as the solar system of coordinates. In this system the photoionization intensity is constant with time in every point of the $F$-layer during the quiet conditions and if its seasonal change is not taken into account. It depends from the spatial coordinates (the latitude, the height) and from the local time (LT), that is from the conditions of the illumination in every point. Thus, the temporal dependence $f \circ F 2$ from the solar local time in the solar coordinate system turns to the spatial dependence from the azimutal coordinate LT.

The observatory motion relatively this irregular $F$-layer during the Earth rotation shows itself as the diurnal changes of the critical frequency $f \circ F 2$ controlled by the solar local time. If in the solar coordinate system the ionization distribution does not change in time, then the diurnal behaviour $f \circ F 2$ will be the same at all stations with the identical latitude. If the ionization changes in time, then the longitudinal variations of the diurnal behaviour $f \mathrm{o} F 2$ should be observed, and then it is logically to speak about UT-control of the ionosphere.

As the illustration, Fig. 1 shows the magnitudes of $f \circ F 2$ as a function of universal time in the solar coordinate system in the points where LT are equal $06,12,18$ and 24 hours. These data were obtained for the stations with the latitudes in the range from $40^{\circ} \mathrm{N}$ to $57^{\circ} \mathrm{N}$ (see Table 1) for September 1964. For $j$-station the universal time of the passage through the point LT is defined by the expression

$$
(\mathrm{UT})_{j}=\mathrm{LT}-\lambda_{j} / 15 \text {, }
$$

here $\lambda_{j}$ is the east longitude in degrees, UT and LT are measured in hours. In this studying the median magnitudes $f \circ F 2$ for one month were used. The scatter of points may be due to the large range of latitudes, therefore it is difficult to detect the $f \circ F 2$-dependence at the universal time. Besides, the stations are nonuniformly distributed: there are many stations in Europe and they are absent in oceans. To compare the dependences for the different values LT, we have used their approximation by the trigonometric 

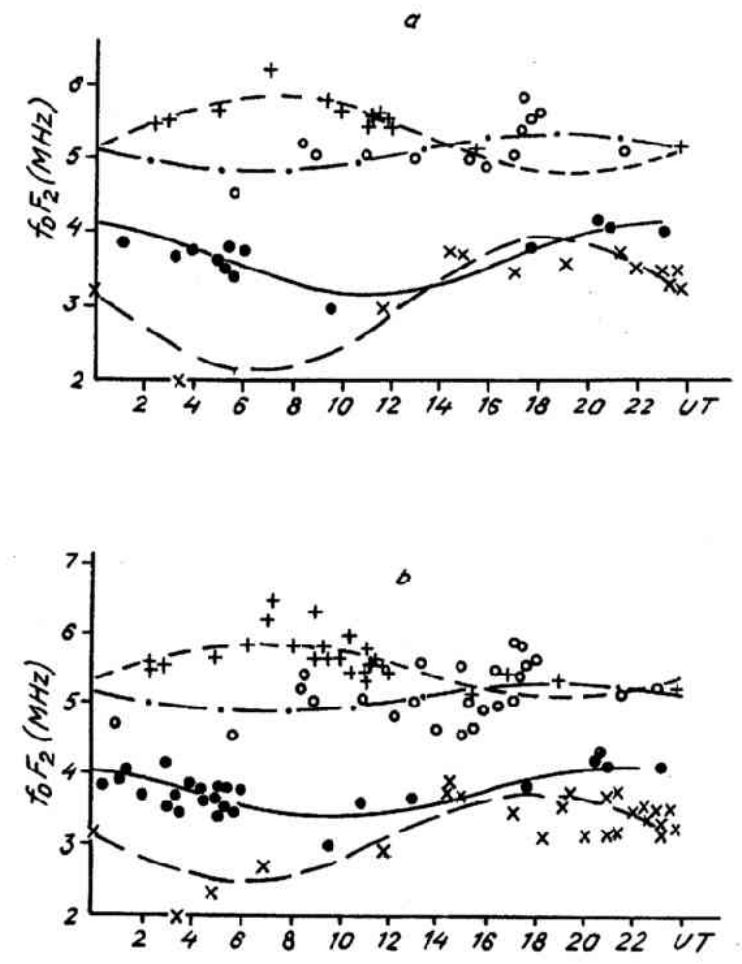

Fig. 1. Approximation $(M=1)$ of the F2-layer critical frequency as a function of the universal time in the solar system of coordinates for 13 stations (a) and for 26 stations (b). The following designations are used for magnitudes of $f \circ F 2$ : $\bullet$ and - at $6 \mathrm{LT},+$ and $-\ldots$ at $12 \mathrm{LT}, \mathrm{O}$ and —.- - at $18 \mathrm{LT}, \times$ and — — at $24 \mathrm{LT}$.

functions in the form

$$
f(t)=a_{0}+\sum_{k=1}^{M}\left(a_{k} \sin \frac{2 \pi k}{T} t+b_{k} \cos \frac{2 \pi k}{T} t\right), \quad T=24 \text { hours. }
$$

The number $M$ of the harmonics which can be used for the approximation is defined by the number of observatories $N$ and their distribution on the longitude. It has to be controlled by the stability of the used approximation with changing of value $M$ and the set of the stations. Figure 1 shows the curves for $M=1$ (the approximation by the diurnal harmonics). Note that in the solar coordinate system the diurnal variations of the ionospheric $F 2$-layer critical frequency as a function of the universal time UT take place in the phase or in the antiphase for the different values of LT. On the day side of the Earth the value $f \circ F 2$ for $12 \mathrm{LT}$ has a maximum around $07 \mathrm{UT}$ and a minimum around $19 \mathrm{UT}$. On the night side the value $f \circ F 2$ for 24 LT has a minimum around 07 UT and a maximum around 19 UT. This result confirms our representation about the existence of the universal ionospheric variation. Certainly, the phenomenon is more complicated and for comparison Fig. 2 shows the approximation of the same data for value $M=2$ that is considering the semidiurnal harmonic. The UT-variation of $f \circ F 2$ is clearly visible here only in the first half-day. It will be showed further that the behaviour of the curves in Fig. 2 can be explained in the terms of the universal variation.

Of large interest are the values $f_{0} F 2$ characterizing their spatial distribution in the solar coordinate system. The local time LT is used as the azimuth coordinate. For the moment of the universal time UT, 
Table 1. Station's list of vertical sounding of the ionosphere.

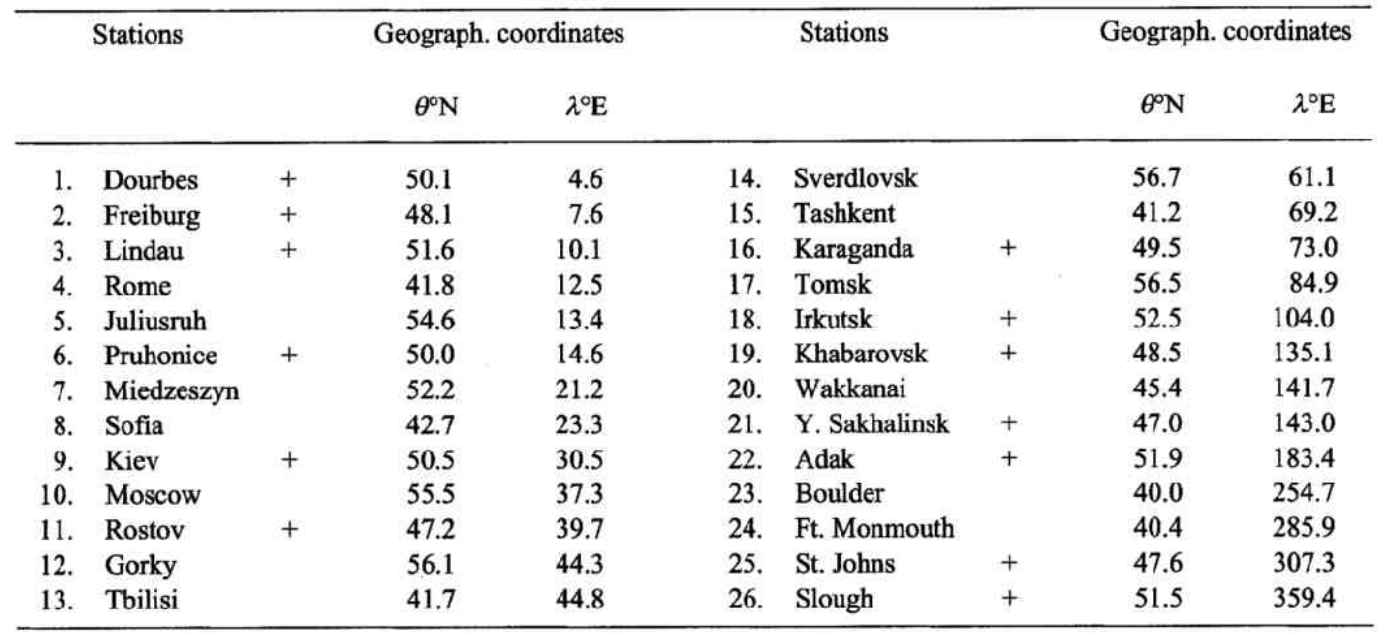

+ marks 13 stations which are in the more narrow interval of the latitudes: from $47.0^{\circ} \mathrm{N}$ till $52.5^{\circ} \mathrm{N}$.
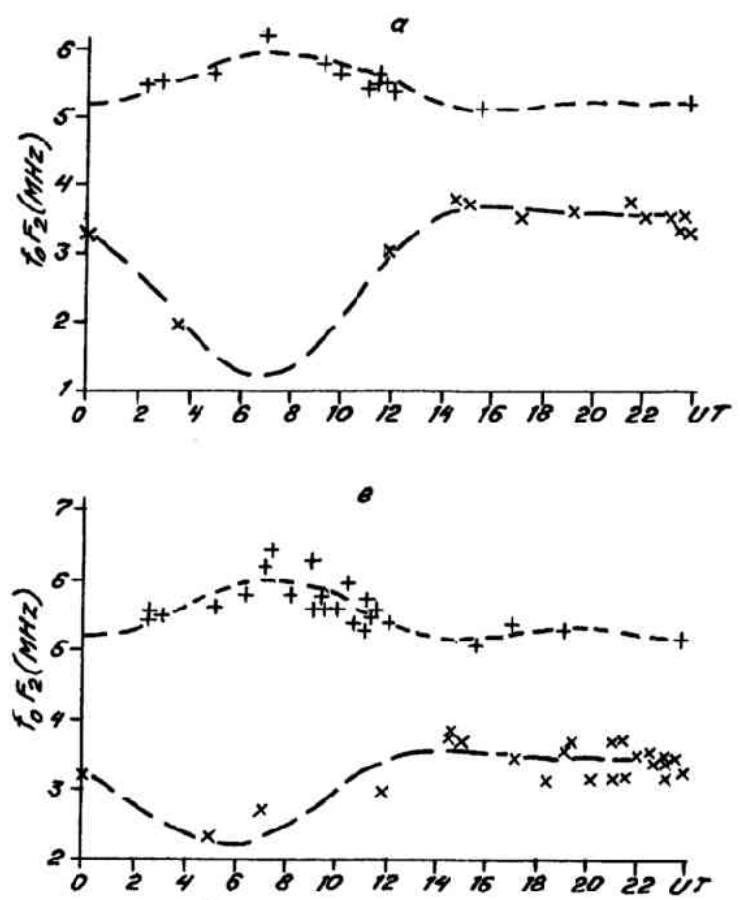

Fig. 2. As in Fig. 1, but $M=2$. 


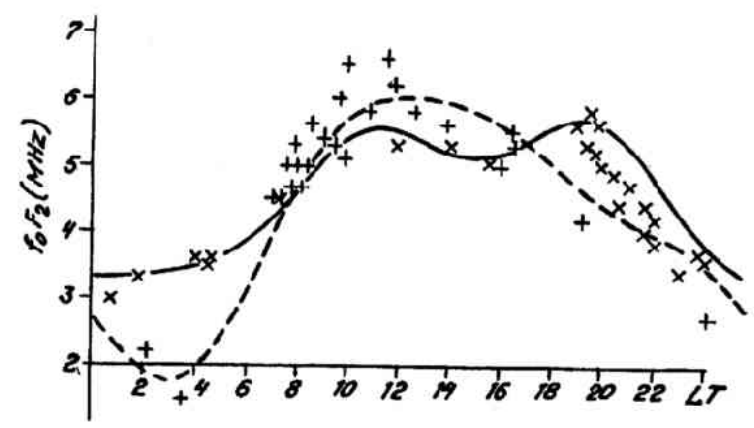

Fig. 3. Spatial distribution of $f \circ F 2$ as a function of the local time in the solar system of coordinates for 26 stations and for $M=$ 3. The following designations are used for magnitudes of $f_{\mathrm{oF}} \mathrm{F}+$ and - - at 7 UT, $\times$ and - at 19 UT.

$j$-station is placed in the point with $(\mathrm{LT})_{j}$ of the solar coordinate system:

$$
(\mathrm{LT})_{j}=\mathrm{UT}+\lambda_{j} / 15
$$

Figure 3 shows for 07 UT and 19 UT the results of the approximation of September 1964 data by means of the first three harmonics. The difference of these curves displays the amplitude distribution of the universal ionospheric variation in the night, day and the sunrise-sunset sectors. Note once more that distinction of the universal variation of the ionospheric parameters is the essential interest for the definition of the physical factors controlling the diurnal ionospheric variation.

\section{Matrix Representation of Geophysical Field}

To determine the phase of the universal variation and the spatial distribution of its amplitude, it is necessary to separate the complicated summary initial fluctuation on the components with the different phase delays. Such problem can be solved by the harmonic analysis method. The complicated summary initial fluctuation shows itself as the longitudinal change of its phase and amplitude characteristics. To clear up in detail the peculiarities of the diurnal variations, it is necessary to do the comparative harmonic analysis of the data for the stations with near latitude.

Consider the common properties of the harmonic concept as the method for the spatial-temporal description of the $f \circ F 2$ diurnal behaviour. The longitudinal-temporal variation of $f \circ F 2$ for the quiet conditions without counting its latitudinal distribution can be presented in the common form:

$$
v(t, \lambda)=\sum_{n, m} V_{n, m} \exp \left\{-2 \pi i\left(\frac{n t}{T}+\frac{m \lambda}{360}\right)\right\} .
$$

Here the geographical coordinate system is used, $\lambda$ is the east longitude in degrees, $t$ is the universal time in hours, $T$ is equal to 24 hours for the diurnal variation, $V_{n, m}$ are the amplitudes of the corresponding harmonic component with period $T / n$ in time and $360 \% \mathrm{~m}$ in longitude. We have taken into account in the expression (4) that $v(t, \lambda)$ is the periodic function in time and in longitude.

In the geographical coordinate system the Sun "rotates" around the Earth from east to west with 24 hours period causing the variation of $f_{\mathrm{o}} F 2$. If this variation were controlled only by the electromagnetic radiation of the Sun, then it should be described by the function which does not change its form with longitude. Only the terms with $m=n$ satisfy such requirement. To understand the role of the another terms 
in (4), we write down the harmonic amplitudes $V_{n, m}$ in the form of the infinite matrix:

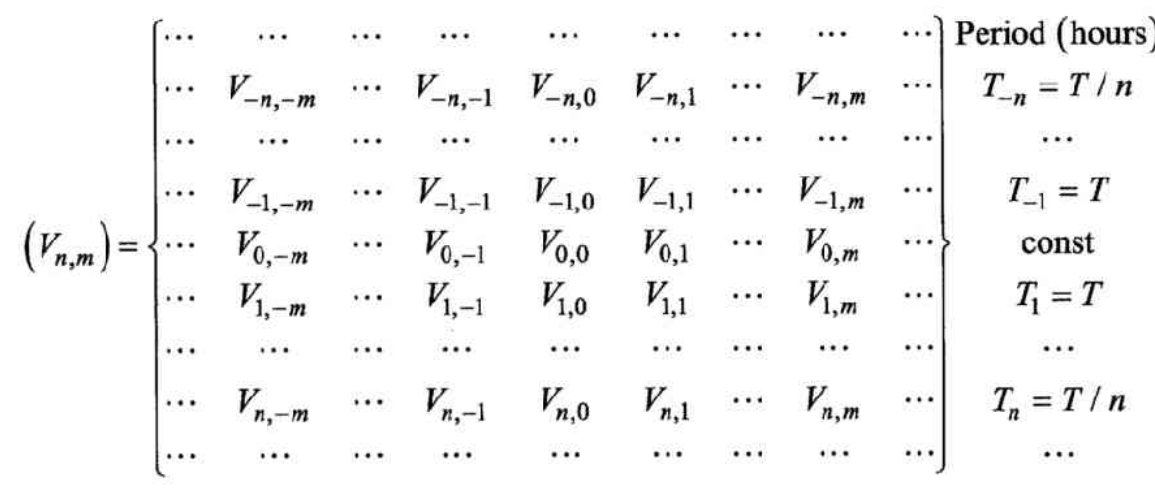

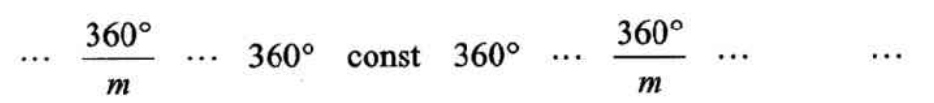

Matrix of the complex amplitudes of the geophysical field.

As is seen from the expr. (4) and(5), the elements of matrix disposed along the maindiagonal $(m=n)$ describe the wave component of the arbitrary form which moves without the distortions from east into west following by the Sun with the velocity equal one revolution per 24 hours. This component controlled by the solar local time can be called as the local solar variation. The elements of matrix disposed along the second main diagonal $(m=-n)$ describe the wave which moves from west to east with the same velocity. The terms united by another diagonals going across the matrix centre $V_{0,0}$ and the element $V_{n, m}$ describe the west and east rotation around the Earth with the velocity $\Delta \lambda \Delta t=-360 \mathrm{n} / \mathrm{Tm}$ degrees in hour (minus corresponds to the rotation from east to west). The greater the angle of the diagonal inclination is different from the main diagonal one, the greater the velocity of the wave moving is different from the diurnal one equal $15 \%$ hour.

The diagonals near the central horizontal line of matrix $(|m|>|n|)$ correspond to the slowly drifting modes. In doing so, the central horizontal line itself describes the irregular in longitude distribution of the investigated geophysical parameter. This component does not change in time and it has been assigned the meaning of the diurnal longitudinal inhomogeneity.

The diagonals near the central vertical column of matrix $(|m|<|n|)$ correspond to the modes which move with velocity greater the diurnal one. The central column describes the uniform in longitude mode having the arbitrary dependence at the universal time. This mode fluctuates with the united on the Earth phase and it was studied by us earlier (Kuznetsov et al., 1990). It can be called as the homogeneous universal variation. We are reminded that we study the quiet steady-state conditions and all variations are periodic in longitude and in time.

As $v(t, \lambda)$ is the real value, it follows that $v^{*}(t, \lambda)=v(t, \lambda)$ and

$$
v^{*}(t, \lambda)=\sum_{n, m} V_{n, m}^{*} e^{2 \pi i\left(\frac{n t}{T}+\frac{m \lambda}{360}\right)}=\sum_{n, m} V_{n, m} e^{-2 \pi i\left(\frac{n t}{T}+\frac{m \lambda}{360}\right)}
$$

and hence $V_{-n,-m}^{*}=V_{n, m}$. Therefore only one-half the matrix (5) elements is independent (for example, at $m \geqslant 0$ ) and the second half the matrix is obtained by the complex conjugation. Using the real functions, 
it is possible to describe the motion of some component with order $(n, m)$ to west in the following form:

$$
f_{n, m}(t, \lambda)=\frac{1}{2} A_{n, m} \cos \left[2 \pi\left(\frac{n t}{T}+\frac{m \lambda}{360}\right)-\varphi_{n, m}\right], \quad n, m \geqslant 0,
$$

and to east in the form:

$$
f_{-n, m}(t, \lambda)=\frac{1}{2} A_{-n, m} \cos \left[2 \pi\left(\frac{n t}{T}-\frac{m \lambda}{360}\right)+\varphi_{-n, m}\right], \quad n, m \geqslant 0 .
$$

Here $A_{n, m}$ and $\varphi_{n, m}$ are the amplitude and phase of the element $V_{n, m}=A_{n, m} e^{i \varphi_{n, m}}$. The elements $V_{n, m}$ and $V_{-n, m}$ are independent and their amplitudes are not equal: $V_{n, m} \neq V_{-n, m}$. Thus there are two running waves: to east and to west. However in the case when

$$
A_{n, m}=A_{-n, m},
$$

one standing wave is formed which is obtained by summing up the expressions (7) and (8):

$$
g_{n, m}(t, \lambda)=A_{n, m} \cos \left[2 \pi \frac{n t}{T}-\frac{\varphi_{n, m}-\varphi_{-n, m}}{2}\right] \cos \left[2 \pi \frac{m \lambda}{360}-\frac{\varphi_{n, m}+\varphi_{-n, m}}{2}\right]
$$

This variation can be called as $n$-harmonic of the inhomogeneous universal variation of order $m$ on the longitude. When the condition (9) is fulfilled, the matrix of the complex amplitudes $V_{n, m}$ has the higher degree of symmetry. The expression (10) does not depend on the sign " $n$ " in view of (9). If we introduce the new phase (the symmetrical phase $\psi_{n, m}^{+}$and the antisymmetrical one $\psi_{n, m}^{-}$):

$$
\psi_{n, m}^{+}=\frac{\varphi_{n, m}+\varphi_{-n, m}}{2}, \quad \psi_{n, m}^{-}=\frac{\varphi_{n, m}-\varphi_{-n, m}}{2}
$$

then one can see that $\psi_{n, m}^{+}$defines the spatial phase of the variation (10) and $\psi_{n, m}^{-}$defines the temporal phase of (10). Using (11), we write the expression of the considered component of the universal variation (that is $n$-harmonic of order $m$ on the longitude) in the form:

$$
g_{n, m}(t, \lambda)=A_{n, m} \cos \left[2 \pi \frac{n t}{T}-\psi_{n, m}^{-}\right] \cos \left[2 \pi \frac{m \lambda}{360}-\psi_{n, m}^{+}\right]
$$

From here, as the special case, the expressions are obtained for the harmonic components namely the diurnal longitudinal inhomogeneity $(n=0)$ or the homogeneous universal variation $(m=0)$. If we sum up the expressions of type (12), it is possible to come to the notion of the generalized universal variation.

Thus, the symmetry degree of matrix (5) is interconnected with the nature of the spatial-temporal distribution of the considered geophysical parameter. In particular, for the critical frequency of the ionosphere $f \mathrm{o} F 2$, the condition (9) is not fulfilled for the elements of the main diagonals. Really, the elements $A_{n, n}$ are greater than $A_{-n, n}$ as it is known that the running to west wave (the local solar variation $\left.f_{\mathrm{o}} F 2\right)$ is prevailing. However, it is unlikely possible to neglect the contribution of the corresponding universal component (for which $\delta A_{n, m}=\delta A_{-n, m}$ ) without the detailed analysis. 


\section{Model of the Description of F2-Layer Critical Frequency}

Of some interest is the problem about the relation between the running and standing waves in the field of the geophysical parameter. This relation is determined, firstly, by the form of the observed longitudinal variations of the diurnal behaviour of the geophysical parameter and, secondly, by the nature of these variations.

This problem can be studied if we will find the matrix representation of the field in the form (5) using the available experimental data. The conditions for the unambiguous restoration of the harmonic components of the complex field impose the certain requirements on the data. We suppose that as usually the spectra are limited from above. According to Kotelnikov's theorem, it is necessary to have at least two measurements on the investigated wavelength. It determines the highest temporal and spatial harmonics. The lower harmonics which can be restored are determined by the interval of the measurement series in time and in space.

As noted above, the elements on the main diagonal of matrix (5) are dominated in this case, they describe the local solar variation. The problem of the choice of another elements of the matrix representation for describing of the diurnal variation is rather complicated. On the one hand, it must be solved by choosing the most steady representation on all available set of the experimental data. On the other hand, it can be made with the help of the additional physical requirements to the separated components of the diurnal variations.

We proceed from the assumption that the diurnal component exists which changes synchronously on all Earth that is the universal variation $f \circ F 2$. Then the temporal phases of the harmonic components $\psi_{n, m}^{-}$ in (12) do not depend on the order " $m$ " $\left(\psi_{n, m}^{-} \equiv \psi_{n}\right)$. In other words, under the assumption that they change synchronously in time, it is possible to write down $n$-harmonic of the universal variation in the following form:

$$
\begin{aligned}
g_{n}(t, \lambda) & =\sum_{m} g_{n, m}(t, \lambda)=\cos \left(2 \pi \frac{n t}{T}-\psi_{n}\right) \sum_{m} A_{n, m} \cos \left(2 \pi \frac{m \lambda}{360}-\psi_{n, m}^{+}\right) \\
& =G_{n}(\lambda) \cos \left(2 \pi \frac{n t}{T}-\psi_{n}\right) .
\end{aligned}
$$

Using (13) and (6), we obtain the representation of diurnal variation of $f \circ F 2$ in such form (for $n$ harmonic):

$$
v_{n}(t, \lambda)=f_{n}(t, \lambda)+g_{n}(t, \lambda)=A_{n} \cos \left[2 \pi n\left(\frac{t}{T}+\frac{\lambda}{360}\right)-\varphi_{n}\right]+G_{n}(\lambda) \cos \left(2 \pi n \frac{t}{T}-\psi_{n}\right)
$$

Returning to the complex amplitudes, we obtain

$$
V_{n}(\lambda)=F_{n} \exp \left(-2 \pi i \frac{n \lambda}{360}\right)+G_{n}(\lambda) \exp \left(i \psi_{n}\right)
$$

Here $V_{n}(\lambda)$ is the complex amplitude of $n$-harmonic of the diurnal variation on the longitude $\lambda, A_{n}$ and $\varphi_{n}$ are the amplitude and phase of the local solar variation, $F_{n}=A_{n} e^{i \varphi_{n}}, G_{n}(\lambda)$ is real amplitude on the longitude $\lambda$ and $\psi_{n}$ is the phase of the universal variation. All phases in (15) are presented at UT. The problem consists in finding the constants $A_{n}, \varphi_{n}, \psi_{n}$ and the function $G_{n}(\lambda)$ which give the best and most steady approximation of the experimental data. 
Note that we do not consider the latitudinal dependence of the investigated geophysical parameter. However the initial data are taken for the stations in some latitudinal interval. Therefore the expression (15) can be considered only as zeroth approximation. To take into account the scatter of stations on the latitude, it is possible to use the evident generalization:

$$
F_{n}(\theta)=F_{n 0}+F_{n 1} \frac{\theta-\theta_{0}}{\theta_{0}}+\cdots, \quad G_{n}(\lambda, \theta)=G_{n 0}(\lambda)+G_{n 1}(\lambda) \frac{\theta-\theta_{0}}{\theta_{0}}+\cdots
$$

Here $\theta$ is a latitude, $\theta_{0}$ is its average magnitude for the taken interval of stations. In this case the number of the unknown values increases therefore the greater number of stations is necessary for solving the problem. However, because of the absence of the necessary number of stations, in this paper we have restricted only the consideration of zeroth approximation.

\section{Method of the Definition of the Model Parameters}

To find the unknowns in (15), we use the methods applied for the solution of the overdetermined system of equations. Let the number of stations is equal $N$. Using the method of the fast Fourier-series in the complex form, we calculate for $j$-station the $n$-harmonic of the median diurnal behaviour $W_{n j}=$ $W_{1 n j}+i W_{2 n j}$. Then the problem can be solved by minimizing the functional $S$ :

$$
S=\sum_{j=1}^{N}\left|V_{n}\left(\lambda_{j}\right)-W_{n j}\right|^{2}
$$

Here $\lambda_{j}$ is the longitude of $j$-station. Instead the experimental amplitudes $W_{n j}$ for approximation of their longitudinal dependence we use trigonometric functions up to $M$-order $W_{n}(\lambda)$

$$
\begin{aligned}
& \operatorname{Re} W_{n}(\lambda)=W_{1 n}(\lambda)=b_{10}+\sum_{k=1}^{M}\left[a_{1 k} \sin \delta_{k}(\lambda)+b_{1 k} \cos \delta_{k}(\lambda)\right], \\
& \operatorname{Im} W_{n}(\lambda)=W_{2 n}(\lambda)=b_{20}+\sum_{k=1}^{M}\left[a_{2 k} \sin \delta_{k}(\lambda)+b_{2 k} \cos \delta_{k}(\lambda)\right], \\
& \delta_{k}(\lambda)=2 \pi k \lambda / 360 .
\end{aligned}
$$

All further calculations are made only for $n$-th harmonic. The coefficients $a_{1 k}, b_{1 k}, a_{2 k}, b_{2 k}$ are found as usually by minimizing the expression for $S_{1,2}$ :

$$
S_{1}=\sum_{j=1}^{N}\left[W_{1 n}\left(\lambda_{j}\right)-W_{1 n j}\right]^{2}, \quad S_{2}=\sum_{j=1}^{N}\left[W_{2 n}\left(\lambda_{j}\right)-W_{2 n j}\right]^{2}
$$

The analogous trigonometric representation can be written for the universal variation amplitude $S_{n}(\lambda)$ :

$$
G_{n}(\lambda)=b_{0}+\sum_{k=1}^{M}\left[a_{k} \sin \delta_{k}(\lambda)+b_{k} \cos \delta_{k}(\lambda)\right]
$$

It follows from (17) that the problem can be solved when suggesting the total coincidence of the 
approximation (15) and (18):

$$
V_{n}(\lambda)=W_{n}(\lambda)
$$

Then from (15), (18), (20) and (21) may write the condition of such coincidence in the form:

$$
\begin{array}{cl}
b_{k} \cos \psi_{n}=b_{1 k}, \quad b_{k} \sin \psi_{n}=b_{2 k}, \\
a_{k} \cos \psi_{n}=a_{1 k}, \quad a_{k} \sin \psi_{n}=a_{2 k}, \quad(k \neq n) \\
b_{n} \cos \psi_{n}+A_{n} \cos \varphi_{n}=b_{1 n}, \quad b_{n} \sin \psi_{n}+A_{n} \sin \varphi_{n}=b_{2 n}, \\
a_{n} \cos \psi_{n}+A_{n} \sin \varphi_{n}=a_{1 n}, \quad a_{n} \sin \psi_{n}-A_{n} \cos \varphi_{n}=a_{2 n}, \quad(k=n) .
\end{array}
$$

It follows that the conditions (22) can be not fulfilled, since in general case $b_{1 k} / a_{1 k} \neq b_{2 k} / a_{2 k}$. Therefore the relations (22) will be roughly fulfilled and the phase $\psi_{n}$ must be obtained by means of the minimization of the functional $S$ from (17). To define the constants $a_{n}, b_{n}, \varphi_{n}$ and $A_{n}$ the system (23) can be used, if writing it in another way:

$$
\begin{gathered}
a_{n}=a_{1 n} \cos \psi_{n}+a_{2 n} \sin \psi_{n}+b_{1 n} \sin \psi_{n}-b_{2 n} \cos \psi_{n}, \\
b_{n}=a_{2 n} \cos \psi_{n}-a_{1 n} \sin \psi_{n}+b_{1 n} \cos \psi_{n}+b_{2 n} \sin \psi_{n}, \\
A_{n} \cos \varphi_{n}=\cos \psi_{n}\left(a_{1 n} \sin \psi_{n}-a_{2 n} \cos \psi_{n}\right)+\sin \psi_{n}\left(b_{1 n} \sin \psi_{n}-b_{2 n} \cos \psi_{n}\right) \\
A_{n} \sin \varphi_{n}=\sin \psi_{n}\left(a_{1 n} \sin \psi_{n}-a_{2 n} \cos \psi_{n}\right)-\cos \psi_{n}\left(b_{1 n} \sin \psi_{n}-b_{2 n} \cos \psi_{n}\right) .
\end{gathered}
$$

Then one can see that the expressions (23) are exactly fulfilled for any phase $\psi_{n}$. In doing so, the expressions (25) define the amplitude $A_{n}$ and the phase $\varphi_{n}$ of the local solar variation and the expressions (24) define the amplitude $G_{n}(\lambda)$ of the universal variation. The phase $\psi_{n}$ of the universal variation becomes the parameter defining the value of functional $S\left(\psi_{n}\right)$ in (17). If fulfilling (23), it is possible to write (17) in following form:

$$
\begin{gathered}
S\left(\psi_{n}\right)=\sum_{j=1}^{N}\left\{\left(b_{0} \cos \psi_{n}-b_{10}\right)+\sum_{\substack{k=1 \\
k \neq n}}^{M}\left[\left(a_{k} \cos \psi_{n}-a_{1 k}\right) \sin \delta_{k}^{j}+\left(b_{k} \cos \psi_{n}-b_{1 k}\right) \cos \delta_{k}^{j}\right]\right\}^{2} \\
+\sum_{j=1}^{N}\left\{\left(b_{0} \sin \psi_{n}-b_{20}\right)+\sum_{\substack{k=1 \\
k \neq n}}^{M}\left[\left(a_{k} \sin \psi_{n}-a_{2 k}\right) \sin \delta_{k}^{j}+\left(b_{k} \sin \psi_{n}-b_{2 k}\right) \cos \delta_{k}^{j}\right]\right\}^{2}, \\
\delta_{k}^{j}=\delta_{k}\left(\lambda_{j}\right)=2 \pi k \lambda_{j} / 360 .
\end{gathered}
$$


One can see from (26) that the minimization of the functional $S\left(\psi_{n}\right)$ brings into the best possible fulfilment of the expressions (22) and hence (21). Thus it brings into the best coincidence of the trigonometric approximations (15) and (18) that is the model and the experiment.

The further calculations are made by the usual way. The set of equations for the calculation of unknown coefficients $b_{k}$ and $a_{k}$ is obtained in following form:

$$
\frac{\partial S}{\partial b_{0}}=0, \quad \frac{\partial S}{\partial a_{k}}=0, \quad \frac{\partial S}{\partial b_{k}}=0, \quad k=1, \ldots, M, \quad k \neq n .
$$

The expressions analogous (24) are found from (27) for $k \neq n$ and it is easy to determine the phase value $\psi_{n}$ for which the functional $S\left(\psi_{n}\right)$ is minimal. This procedure is performed numerically, therefore we present in the obvious form only the set (27) for $k \neq n$ :

$$
\left(\begin{array}{cccccc}
\vec{S}_{1} \cdot \vec{S}_{1} & \vec{S}_{1} \cdot \vec{C}_{1} & \cdots & \vec{S}_{1} \cdot \vec{S}_{M} & \vec{S}_{1} \cdot \vec{C}_{M} & \vec{S}_{1} \cdot \vec{C}_{0} \\
\vec{C}_{1} \cdot \vec{S}_{1} & \vec{C}_{1} \cdot \vec{C}_{1} & \cdots & \vec{C}_{1} \cdot \vec{S}_{M} & \vec{C}_{1} \cdot \vec{C}_{M} & \vec{C}_{1} \cdot \vec{C}_{0} \\
\cdots & \cdots & \cdots & \cdots & \cdots & \cdots \\
\vec{S}_{M} \cdot \vec{S}_{1} & \vec{S}_{M} \cdot \vec{C}_{1} & \cdots & \vec{S}_{M} \cdot \vec{S}_{M} & \vec{S}_{M} \cdot \vec{C}_{M} & \vec{S}_{M} \cdot \vec{C}_{0} \\
\vec{C}_{M} \cdot \vec{S}_{1} & \vec{C}_{M} \cdot \vec{C}_{1} & \cdots & \vec{C}_{M} \cdot \vec{S}_{M} & \vec{C}_{M} \cdot \vec{C}_{M} & \vec{C}_{M} \cdot \vec{C}_{0} \\
\vec{C}_{0} \cdot \vec{S}_{1} & \vec{C}_{0} \cdot \vec{C}_{1} & \cdots & \vec{C}_{0} \cdot \vec{S}_{M} & \vec{C}_{0} \cdot \vec{C}_{M} & \vec{C}_{0} \cdot \vec{C}_{0}
\end{array}\right) \times\left(\begin{array}{c}
a_{1} \\
b_{1} \\
\cdots \\
a_{M} \\
b_{M} \\
b_{0}
\end{array}\right)=\left(\begin{array}{c}
\vec{V} \cdot \vec{S}_{1} \\
\vec{V} \cdot \vec{C}_{1} \\
\cdots \\
\vec{V} \cdot \vec{S}_{M} \\
\vec{V} \cdot \vec{C}_{M} \\
\vec{V} \cdot \vec{C}_{0}
\end{array}\right)
$$

Here for convenience $N$-vectors are introduced which are the magnitudes of the used values for $N$ stations and here the usual scalar product of such vectors is used. In particular, in (28) the designations are introduced:

$$
\begin{aligned}
& \vec{S}_{k}=\left\{\sin \delta_{k}\left(\lambda_{1}\right), \ldots, \sin \delta_{k}\left(\lambda_{N}\right)\right\}, \quad k=1, \ldots, M, \\
& \vec{C}_{k}=\left\{\cos \delta_{k}\left(\lambda_{1}\right), \ldots, \cos \delta_{k}\left(\lambda_{N}\right)\right\}, \quad k=1, \ldots, M, \\
& \vec{C}_{0}=\{1,1,1, \ldots, 1,1,1\}, \quad k=0, \\
& \vec{V}=\left\{\bar{V}_{1}\left(\lambda_{1}\right) \cos \psi_{n}+\bar{V}_{2}\left(\lambda_{1}\right) \sin \psi_{n}, \ldots, \bar{V}_{1}\left(\lambda_{N}\right) \cos \psi_{n}+\bar{V}_{2}\left(\lambda_{N}\right) \sin \psi_{n}\right\}, \\
& \bar{V}_{1,2}\left(\lambda_{j}\right)=b_{10,20}+\sum_{\substack{k=1 \\
k \neq n}}^{M}\left[a_{1 k, 2 k} \sin \delta_{k}\left(\lambda_{j}\right)+b_{1 k, 2 k} \cos \delta_{k}\left(\lambda_{j}\right)\right] .
\end{aligned}
$$

Solving the set (28) for the given value $\psi_{n}$, we find the coefficients $a_{k}$ and $b_{k}$ for $k \neq n$ and from (24) we deduce also $a_{n}$ and $b_{n}$. In doing so the functional value $S\left(\psi_{n}\right)$ is defined in (26) which is further minimized. That is the algorithm of calculation which can be used for the separation of the observed diurnal variation of the ionospheric parameters on the local and universal components.

\section{Calculation Results}

Using this algorithm, we have processed the ionospheric data for the stations which list is presented in Table 1. Figure 4 shows the complex amplitudes $W_{n j}=W_{1 n j}+i W_{2 n j}$ of the diurnal harmonic $f \circ F 2(n=$ 1) at UT calculated according to our scheme and marked by crosses $\left(W_{1 n j}\right.$ at the left and $W_{2 n j}$ at the right 
of Fig. 4). The curve marked by the dashed line presents the spatial trigonometric approximations $W_{1 n}(\lambda)$ and $W_{2 n}(\lambda)$ of the longitudinal distribution of these amplitudes according to (18) for $M=3$.

Figure 5 displays the longitudinal behaviour the modules $\left|W_{n j}\right|$ (a) and the phases $\operatorname{Arg}\left(W_{n j}\right)$ (b) of the same complex amplitudes but at the corresponding solar local time (not at the zone time as it is usually conventional). These magnitudes are marked by crosses. Figure 5 shows also the module $\left|W_{n}(\lambda)\right|(\mathrm{a})$ and the phase $\operatorname{Arg}\left(W_{n}(\lambda)\right)$ (b) of their spatial trigonometric approximations marked by the dashed lines.

It is interesting to note that the maxima of the module $\left|W_{n}(\lambda)\right|$ for the Northern hemisphere are in the longitudinal sectors $50^{\circ}-100^{\circ} \mathrm{E}$ and $250^{\circ}-300^{\circ} \mathrm{E}$ where the magnetic and geomagnetic poles are placed. The first minimum of the phase $\operatorname{Arg}\left(W_{n}(\lambda)\right)$ is also located between $50^{\circ}-100^{\circ} \mathrm{E}$ but the second minimum placed at $230^{\circ} \mathrm{E}$ is offset to the left from the sector $250^{\circ}-300^{\circ} \mathrm{E}$.

This circumstance is not obviously casual and it provides reason enough to say about the geomagnetic control of the ionosphere. Figure 6(b) shows the dependence of the functional $S\left(\psi_{n}\right)$ (see expression (26)) on the universal variation phase $\psi_{n}$. The minimization process is showed here according to the above considered scheme.

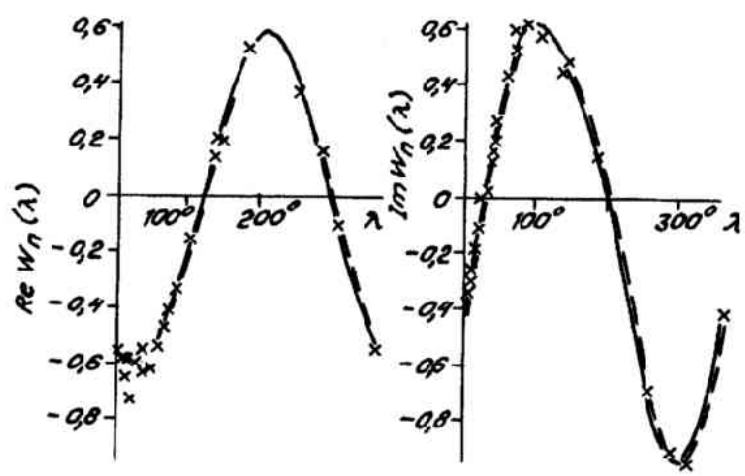

Fig. 4. Longitudinal distribution of the complex amplitudes of the diurnal harmonic of $f \circ F 2$ at universal time for $n=1$. The following designations are used: $\times$ means the complex amplitudes $W_{n j}=W_{1 n j}+i W_{2 n j}$ on the stations: $W_{1 n j}$ at the left and $W_{2 n j}$ at the right, - - means their spatial trigonometric approximations $(18) W_{n}(\lambda)=W_{1 n}(\lambda)+i W_{2 n}(\lambda)$ for $M=3, W_{1 n}(\lambda)$ at the left and $W_{2 n}(\lambda)$ at the right, - displays graphically the model with the universal and local variations.

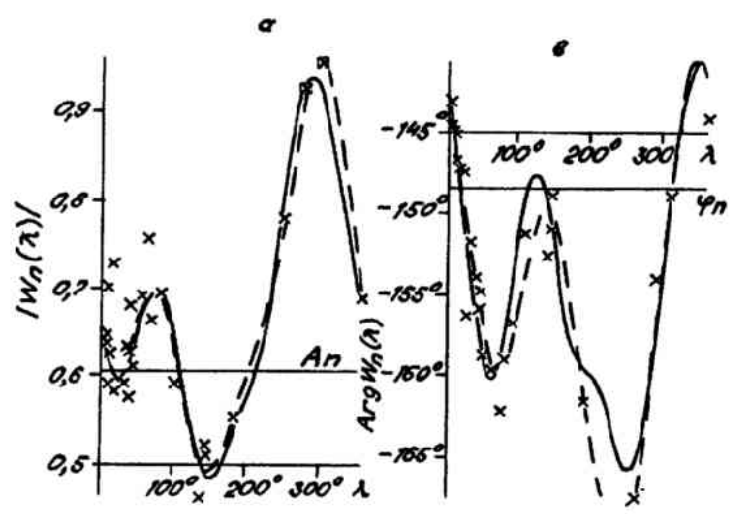

Fig. 5. Longitudinal distribution of the modules (a) and phases (b) of the complex amplitudes of the diurnal harmonic of $f_{0} F 2$ at local time for $n=1$. The following designations are used: $\times$ means the values $\left|W_{n j}\right|$ (a) and $\operatorname{Arg}\left(W_{n j}\right)$ (b) on the stations, -- - means their spatial trigonometric approximations (18) for $M=3,\left|W_{n}\right|(\lambda)$ (a) and $\operatorname{Arg}\left(W_{n}(\lambda)\right)(\mathrm{b}),-$ displays graphically the model with the universal and local variations. 
One minimum of $S\left(\psi_{n}\right)$ is for $\psi_{\min } \approx 260^{\circ}$. Figure 6(a) shows the longitudinal distribution of the universal variation amplitude $G_{n}(\lambda)(20)$ obtained in this case. If the first minimum $S\left(\psi_{n}\right)$ for $\psi_{\min } \approx 80^{\circ}$ is taken into account, then the amplitude distribution changes the sign. Here it should be remembered that the negative amplitude corresponds the change of the universal variation phase on $180^{\circ}$ (the diurnal oscillations in the antiphase). One can see from Fig. 6 that the extreme of the amplitudes $G_{n}(\lambda)$ are placed in the longitudinal sectors $50^{\circ}-100^{\circ} \mathrm{E}$ and $250^{\circ}-300^{\circ} \mathrm{E}$ which contain the agonic line connecting the Canadian and the East-Siberian world magnetic anomalies marked by A (Fig. 7, the arrows show the directions of their secular motion (Hope, 1959)). Figure 8(a) shows the diurnal universal harmonic on the phase at universal time for some longitudes which are marked by the numbers. The phase of the universal variation is to be related to the instants of noon and midnight at these longitudinal sectors. Thus, the diurnal universal variation has the greatest amplitudes at the longitudes $50^{\circ}-100^{\circ} \mathrm{E}$ and $250^{\circ}-300^{\circ} \mathrm{E}$, its change in time at these sectors occurs in antiphase. Besides, the universal variation has the maxima and minima

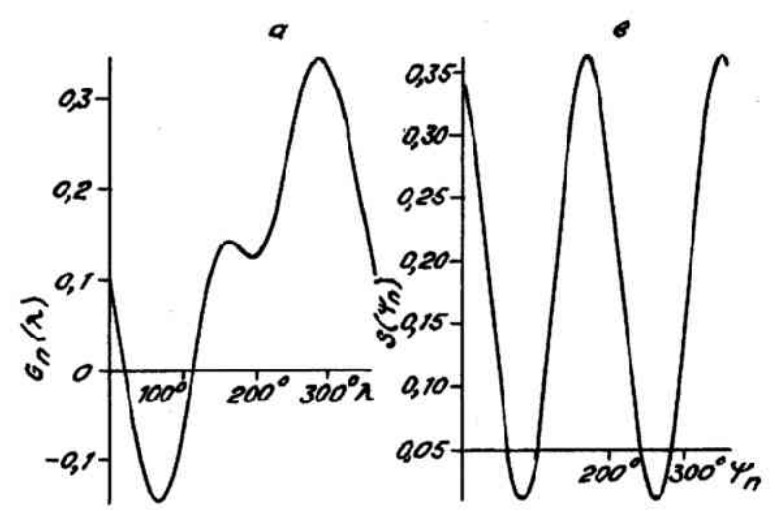

Fig. 6. Longitudinal distribution of the diurnal harmonic amplitude of the universal variation $G_{n}(\lambda)$ (a) and the value of the functional $S\left(\psi_{n}\right)$ versus the universal variation phase $\psi_{n}$ for $n=1$ (b).

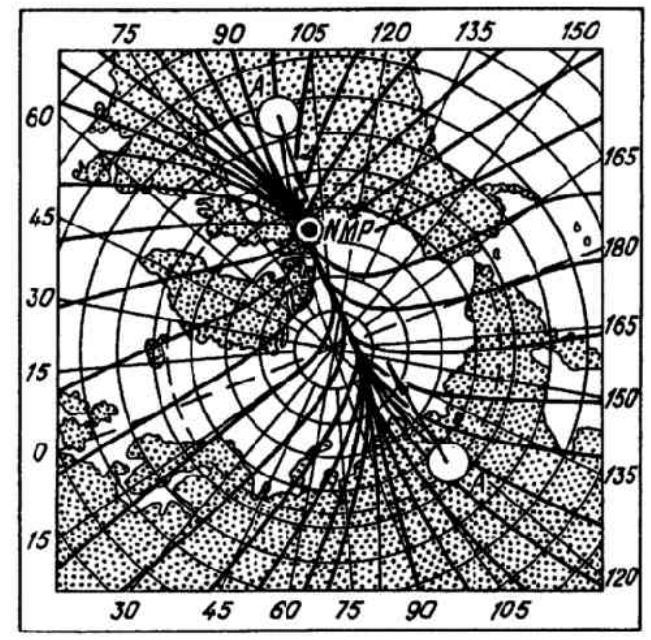

Fig. 7. Map of the magnetic meridians in the Northern hemisphere. The sign $\odot$ marks the Northern magnetic pole, the designations A mark the Canadian and the East-Siberian world magnetic anomalies, the arrows show the directions of their secular motion (Hope, 1959). 


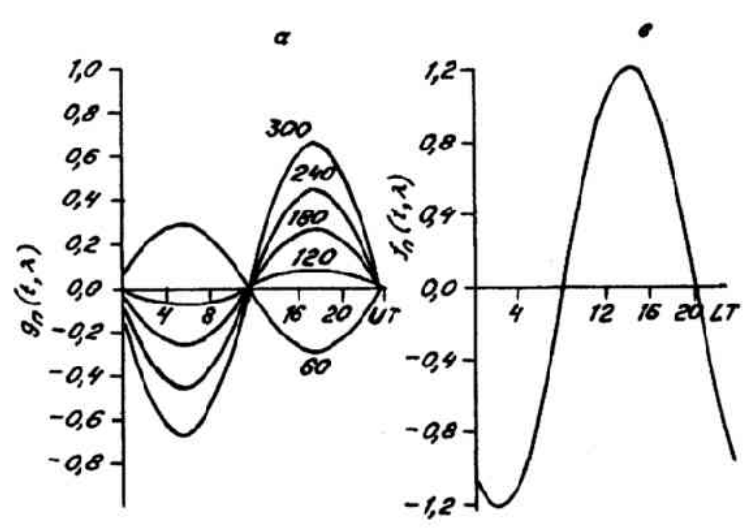

Fig. 8. The form of the universal variation (a) and the local solar variation (b) for the diurnal harmonics. The numbers near the curves of Fig. 8(a) mark the longitudes.

in the instant when these longitudinal sectors go across the direction to the Sun.

The behaviour of $f \mathrm{o} F 2$ becomes clear now depending on the universal time in the solar system of coordinates near the noon (12 LT) and the midnight (24 LT) as illustrated in Figs. 1 and 2. In the solar system of coordinates the diurnal universal variation $f \mathrm{o} F 2$ takes part in the daily rotation together the Earth. In this case the first longitudinal sector $\left(50^{\circ}-100^{\circ} \mathrm{E}\right)$ goes across the noon region (12 LT) at the interval 05-09 UT. As one can see from Fig. 8(a), the universal variation at this longitudinal sector has maximum at the same time. As the result of that, in the noon point the value $f 0 F 2$ increases at $06-08$ UT (see Figs. 1 and 2). The second longitudinal sector $\left(250^{\circ}-300^{\circ} \mathrm{E}\right)$ at $06-09 \mathrm{UT}$ goes across the midnight region (24LT), here at this time the universal variation is minimal (see Fig. 8(a)). This causes the minimum in the curves for 24 LT (Figs. 1 and 2).

In twelve hours the longitudinal sectors $50^{\circ}-100^{\circ} \mathrm{E}$ and $250^{\circ}-300^{\circ} \mathrm{E}$ during the daily rotation change places relatively the points with $L T=12$ hours and LT $=24$ hours. In this case the universal variation phase changes sign on contrary one in both sectors (Fig. 8(a)). Thus, in the noon point the value $f \circ F 2$ must again increase and for $24 \mathrm{LT}$ it will decrease. However, it is appeared slightly in the curves of Fig. 2 at 18-20 $\mathrm{UT}$, and in Fig. 1 the contrary change $f_{0} F 2$ is even observed. It can be explained by that here the diurnal harmonic of the universal variation was only taken into account whereas the summary pattern observed in experiment is presented in Figs. 1 and 2. Besides, it should be taken into consideration that the diurnal harmonic of universal variation in the geographical system of coordinates corresponds to the semidiurnal harmonic in the solar system of coordinates. The diurnal harmonic in the solar system is formed by the rotation together with the Earth the ionization distribution which is constant in time but irregular on longitude in the geographical system of coordinates. Such distribution was above called as the diurnal longitudinal inhomogeneity.

Figure 9 shows the temporal behaviour of $f_{\mathrm{o}} F 2$ at UT in the noon (12 LT) and midnight (24 LT) regions in the solar system of coordinates which is caused by the rotation of the diurnal longitudinal inhomogeneity. The approximation by means of the diurnal harmonics is given by the dashed curve for 12 LT and by the long-dashed curve for 24 LT. The designations ( + ) for 12 LT and $(x)$ for 24 LT show the mean-diurnal components for the different stations (zeroth harmonics) but not the summary values of $f \mathrm{oF} 2$ which are shown by $(+)$ and $(x)$ in Figs. 1 and 2 . The diurnal harmonics shown in Fig. 1 are basically conditioned by the rotation of the diurnal longitudinal inhomogeneity. In Fig. 2 the summary influence of two factors is noticeable namely the rotation of the longitudinal inhomogeneity and the diurnal universal variation. At the first half of day at the universal time these two factors act in phase and at second half they act in antiphase. As the result of that we observe the pattern in Fig. 2: the noticeable extreme of 


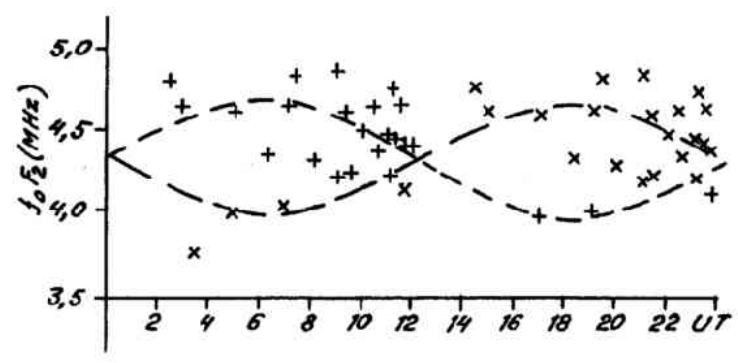

Fig. 9. The influence of the diurnal longitudinal inhomogeneity on the $f \circ F 2$-behaviour in the solar system coordinates for data of 26 stations. The following designations are used for the mean-diurnal components of $f \circ F 2$ (zeroth harmonics): + and -. - at $12 \mathrm{LT}, \times$ and $-\ldots$ at $24 \mathrm{LT}$.

$f \mathrm{oF} 2$ near 07 UT and the less pronounced extreme at $19 \mathrm{UT}$.

Note that in Fig. 9 the constant component of $f_{0} F 2$ (the diurnal longitudinal inhomogeneity) has also the peculiarities in the above-mentioned sectors $50^{\circ}-100^{\circ} \mathrm{E}$ and $250^{\circ}-300^{\circ} \mathrm{E}$ (it should be remarked that for $24 \mathrm{LT}$ the axis of abscissa can be related to the axis of the decreasing longitudes in the interval $\left.360^{\circ}-0^{\circ}\right)$.

Except the characteristics of the universal variation, from (25) the amplitude $A_{n}$ and the phase $\varphi_{n}$ of the local solar variation can be determined. In Fig. 5 these value are marked as $A_{n}$ and $\varphi_{n}$; the solar variation at the solar local time LT is presented in Fig. 8(b).

Thus we have suggested the empirical model for describing the longitudinal effect in $f \circ F 2$. The approximation quality of the longitudinal effect by means of this model is well represented by Figs. 4 and 5. In Fig. 4 the distinction of the initial approximation from the model one is small (here data are presented at UT). The agreement between model and experiment at the local time is seen in Fig. 5. There is good reason to believe that the model in the form of sum of the diurnal universal variation and the local one is well described the longitudinal effect.

\section{Conclusion}

For the analysing of the longitudinal effect of $f_{0} F 2$ by the method described above it is necessary to use the results synchronous ionospheric observations at the stations placed uniformly on the longitude and on given latitude ring. Such data availability has given us the chance to study the peculiarities of the longitudinal-temporal variations of ionospheric parameters. This analysis confirms the conclusion that diurnal longitudinal-temporal variations of the critical frequency $f_{0} F 2$ in the mid-latitudinal ionosphere contain the universal variation. The longitudinal effect in the diurnal behaviour of $f_{0} F 2$ is adequately described by the sum of the variation controlled by the solar local time and the universal variation. The latter is characterized by the united variation of $\mathrm{foF}_{\mathrm{o}}$ at UT at all longitudes of the circle with the given latitude (in the geographical system of coordinates). The temporal dependence of the universal variation is characterized by existence of the extreme at 07 UT and 19 UT. These instants of the time coincide with the noon and the midnight at the solar local time in the longitudinal sectors $50^{\circ}-100^{\circ} \mathrm{E}$ and $250^{\circ}-300^{\circ} \mathrm{E}$ which are connected with the peculiarities of the Earth's magnetic field. The spatial (longitudinal) dependence of the universal variation amplitude is characterized by the extreme in the same longitudinal sectors. These facts anable to say about the existence of the geomagnetic control of the $f \circ F 2$-universal variation.

The authors acknowledge support by Russian Basic Science Foundation grant 94-05-16041-a.

The Editor thanks S. Watanabe and another referee for their assistance in evaluating this paper. 


\section{REFERENCES}

Appleton, E. V., Two anomalies in the ionosphere, Nature, 157, 691, 1946.

Bailey, D. K., The geomagnetic nature of the F2-layer longitude effect, Terr. Magn. Atm. Electr, 53, $35,1948$.

Besprozvannaya, A. S. and L. N. Makarova, UT-control of the configuration of the ionospheric mid-latitude trough, Geomagn. Aeron., 24, 145-146, 1984 (in Russian).

Challinor, R. A., The behaviour of the Arctic F-region in winter, J. Atmos. Terr. Phys., 32, 1959-1963, 1970.

Challinor, R. A. and D. Eccles, Longitudinal variations of the mid-latitude ionosphere produced by neutral-air winds, J. Atmos. Terr. Phys., 33, 363-369, 1971.

Chalmers, J. A., Atmospheric Electricity, Pergamon Press, 1967.

Deminov, M. G. and A. T. Karpachev, The longitudinal effect in the configuration of the ionospheric mid-latitude trough. 1. The localization of the trough, Geomagn. Aeron., 26, 63-68, 1986 (in Russian).

Duncan, R. A., Universal-time control of the Arctic and Antarctic F-region, J. Geophys. Res., 67, 1823-1830, 1962.

Eccles, D., J. W. King, and P. J. Rothwell, Longitudinal variations of the mid-latitude ionosphere produced by neutral-air wind, J. Atmos. Terr. Phys., 33, 371-377, 1971.

Eyfrig, R. W., The effect of the magnetic declination on the F2-layer, Ann. Geophys., 19, 102-107, 1963.

Hope, E. R., Geotectonic of the Arctic ocean and the Great Arctic magnetic Anomaly, J. Geophys. Res., 64, 407-427, 1959.

Hopkins, H. D., R. J. D'Arcy, and J. Sayers, UT-control of the topside polar ionosphere, Planet. Space Sci., 21, 1459-1462, 1973.

Kazimirovsky, E. S., M. P. Rudina, A. I. Pogoreltsev, and G. Ya. Khachikyan, Meteorological effects in the $F 2$-layer of the ionosphere, Geomagn. Aeron., 23, 208-212, 1983 (in Russian).

Kessenikh, V. N. and N. D. Bulatov, Continental effect in the geographical distribution of the F2-layer electron density, Dokl. Akad. Nauk SSSR, 45, 250 256, 1944 (in Russian).

Khachikyan, G. Ya. and M. P. Rudina, Longitudinal effects in the distribution of the critical frequencies of the $F 2$-layer of the ionosphere, Geomagn. Aeron., 19, 232-236, 1979 (in Russian).

King, J. W., H. Kohl, D. M. Preece, and C. Seabrook, An explanation of phenomena occurring in the high-latitude ionosphere at certain Universal Time, J. Atmos. Terr. Phys., 30, 11-23, 1968.

Kneht, R. W., Observations of the ionosphere over the South Geographic Pole, J. Geophys. Res., 64, $1243-1250,1959$.

Kochenova, N. A., Longitudinal variations of the equatorial ionosphere from the satellite's Intercosmos-19 data, Geomagn. Aeron., 27, 142-144, 1987 (in Russian).

Kohnlein, W. and W. J. Raitt, ESRO-1 and ESRO-2: a model of the UT-effect in electron density at middle latitudes of the southern hemisphere, Planet. Space Sci., 26, 1179-1184, 1978.

Kuznetsov, V. V., V. V. Plotkin, I. I. Nesterova, and M. S. Pozdeeva, Universal diurnal variation of $F 2$-layer critical frequency, J. Geomag. Geoelectr., 42, 1237-1240, 1990.

Kuznetsov, V. V., V. V. Plotkin, I. I. Nesterova, and N. I. Izraileva, Universal geomagnetic variation, J. Geomag. Geoelectr., 44, $481-494,1992$.

Kuznetsov, V. V., V. V. Plotkin, I. I. Nesterova, and N. I. Izraileva, Universal variations of ionospheric parameters and the geomagnetic field: theoretical view and evaluations, J. Atmos. Terr. Phys., 55, 1575-1582, 1993.

Maksimova, N. M. and N. K. Osipov, The UT-effects in the polar ionosphere, Geomagn. Aeron., 24, 403-406, 1984 (in Russian).

Osipov, N. K. and N. M. Maksimova, The UT-effects in the variations of the structures of the magnetospheric convection and the polar ionosphere, Geomagn. Aeron., 23, 93-97, 1983 (in Russian).

Pancheva, D. V., Electric field effect on longitudinal variations of $F$ night region, Dokl. Bulgar AN, 39, 65-68, 1986.

Rastogi, R. G., Longitudinal effect in the equatorial F2-region of the ionosphere, J. Atmos. Terr. Phys., 25, 739-742, 1963.

Schweidler, E., Die aufrecterhaltung der elektrischen Ladung der Erde, in Probleme der Kosmischen Physik, edited by C. Jensen and A. Schwassmann, XY, 75 pp., Hamburg, 1932.

Tulunay, Y. J., Global electron density distribution from the Ariel-3 satellite at mid-latitudes during quiet magnetic periods, $J$. Atmos. Terr. Phys., 35, 233-254, 1973. 